\title{
Influência da morfologia de pés e joelhos no equilíbrio durante apoio bipodal
}

\author{
Influence of feet and knees morphology on balance during bipodal stance
}

\author{
Arthur de Sá Ferreira ${ }^{[a]}$, Nárrima de Souza Gave ${ }^{[b]}$, Fabiana Abrahão ${ }^{[c]}$, Julio Guilherme Silva ${ }^{[\mathrm{d}]}$ \\ [a] Fisioterapeuta, Doutor em Ciências em Engenharia Biomédica, professor adjunto do Centro Universitário Augusto Motta, \\ Laboratório de Análise do Movimento Humano, Programa de Pós-Graduação em Ciências da Reabilitação, Centro Universitário \\ Augusto Motta (UNISUAM), Rio de Janeiro, RJ - Brasil, e-mail: arthur_sf@ig.com.br \\ [b] Fisioterapeuta, Pós-Graduada em Biomecânica, Mestranda em Engenharia Biomédica pelo Instituto Alberto Luiz Coimbra de \\ Pós-Graduação e Pesquisa em Engenharia (COPPE/UFRJ), Rio de Janeiro, RJ - Brasil, e-mail: narrimagave@hotmail.com \\ [c] Fisioterapeuta, Doutora em Fisioterapia, professora adjunta do Centro Universitário Augusto Motta, Laboratório de Desempenho \\ Neurofuncional Programa de Pós-Graduação em Ciências da Reabilitação, Centro Universitário Augusto Motta (UNISUAM), \\ Rio de Janeiro, RJ - Brasil, e-mail: fabiana.abrahao@yahoo.com.br \\ [d] Fisioterapeuta, Doutor em Saúde Mental - Aprendizagem Motora, professor adjunto do Centro Universitário Augusto Motta, \\ Laboratório de Análise do Movimento Humano, Programa de Pós-Graduação em Ciências da Reabilitação, Centro Universitário \\ Augusto Motta (UNISUAM), Rio de Janeiro, RJ - Brasil, e-mail: julioguilherme@unisuam.edu.br
}

\section{Resumo}

Introdução: A relação entre o padrão morfológico de pés/joelhos e o equilíbrio é estudada por meio de diversos modelos experimentais. Entretanto, as investigações ainda são controversas, especialmente na comparação estabilométrica dos tipos de joelho em apoio bipodal. Objetivo: Analisar a influência da morfologia de pés e joelhos nos parâmetros estabilométricos de indivíduos saudáveis. Metodologia: Participaram 53 indivíduos hígidos (21 [39,6\%] do sexo masculino, 26,9 \pm 5,2 anos, 68,4 $\pm 17,1 \mathrm{~kg}$, $1,71 \pm 0,09 \mathrm{~m})$. A avaliação morfológica de pés/joelhos foi realizada por meio de avaliação postural pelo método visual não quantitativo, e o resultado foi dado por dois avaliadores simultaneamente. $\mathrm{O}$ protocolo de estabilometria (50 segundos) avaliou as combinações de olhos abertos/fechados com base aberta/fechada. $\mathrm{Na}$ análise estatística foi utilizado o coeficiente de contingência para verificar associação entre os achados morfológicos. As variáveis antropométricas e estabilométricas foram analisados pela ANOVA, com significância de $\mathrm{p}<0,05$. Resultados: Indivíduos com joelho varo apresentaram menor velocidade de oscilação que os classificados como joelho neutro nos protocolos de olhos abertos (joelho varo: $6,86 \pm 3,0410^{-2} \mathrm{~m} / \mathrm{s}$; joelho neutro: $11,61 \pm 6,3610^{-2} \mathrm{~m} / \mathrm{s} ; \mathrm{p}=0,009$ ) e olhos fechados (joelho varo: $6,92 \pm 2,7710^{-2} \mathrm{~m} / \mathrm{s}$; joelho neutro: $\left.11,38 \pm 5,8210^{-2} \mathrm{~m} / \mathrm{s} ; \mathrm{p}=0,007\right)$, ambos em base fechada. Não foram observadas diferenças significativas entre os indivíduos nas variáveis área de oscilação (elipse 
percentil 95) e deslocamento máximo no sentido ântero-posterior. Conclusão: A morfologia do pé não alterou significativamente a velocidade de oscilação, a área de oscilação ou o deslocamento ânteroposterior. A redução da base de suporte reduz a velocidade de oscilação em indivíduos com joelho varo.

Palavras-chave: Postura. Joelho. Pé. Equilíbrio musculosquelético.

\begin{abstract}
Introduction: The relationship between morphological patterns of feet/knees and balance have been studied through several experimental models. However, there is no consensus about knee morphology influences on balance during bipodal stance. Objective: To analyze the influence of morphological types of feet and knees on stabilometric variables of healthy subjects. Methods: Fifty-three asymptomatic subjects were enrolled in this study (21 [39.6\%] male, $26.9 \pm 5.2$ years, $68.4 \pm 17.1 \mathrm{~kg}, 1.71 \pm 0.09 \mathrm{~m})$. Morphologic classification of lower extremity was made through visual inspection by two examiners. Stabilometric protocol was performed (50 seconds) with eyes opened/closed and base opened/closed. Statistical analysis used contingency coefficient to check for association among the morphological findings. Anthropometric and stabilometric variables were analyzed with one-way ANOVA with significant level of $p<0,05$. Results: Subjects with genu varus presented lower oscillation velocity compared to physiological genu valgus at protocols with opened eyes (varus: $6.86 \pm 3.0410^{-2} \mathrm{~m} / \mathrm{s} ;$ physiologic valgus: $11.61 \pm 6.3610^{-2} \mathrm{~m} / \mathrm{s} ; p=0,009$ ) and closed eyes (varus: $6.92 \pm 2.7710^{-2} \mathrm{~m} / \mathrm{s}$; physiologic valgus: $11.38 \pm 5.8210^{-2} \mathrm{~m} / \mathrm{s} ; p=0,007$ ), both with closed base. No significant differences among subjects were observed on variables sway area (95th percentile ellipse) and maximum anterior-posterior displacement. Conclusion: Feet morphology did not change sway velocity, sway area, or anteroposterior displacement. Reduction of the support base diminished sway velocity in subjects with genu varus.
\end{abstract}

Keywords: Posture. Knee. Foot. Musculoskeletal balance.

\title{
Introdução
}

Dificuldades na manutenção do equilíbrio ortostático contribuem para o aumento do risco de quedas. Indivíduos idosos $(1,2)$ ou com desordens do sistema nervoso $(3,4)$ apresentam riscos mais elevados para esse desfecho. A posição ortostática coloca o corpo em equilíbrio postural instável (oscilação postural), por causa da elevada posição do Centro de Gravidade (CG), mantido na reduzida base de suporte através de ajustes contínuos $(5,6)$. O controle postural compreende o processo de percepção e processamento de informações provenientes do sistema visual, somatosensorial e vestibular, e a seleção da resposta motora adequada para manter ou restaurar o equilíbrio postural (7). A avaliação da oscilação postural pode fornecer informações a respeito das variáveis que influenciam o controle postural, contribuindo clinicamente para a redução das quedas e suas lesões associadas.

Testes clínicos (8) podem avaliar como as deficiências no equilíbrio, na força e na resistência musculares afetam as atividades diárias. Entre eles, destacam-se: teste de equilíbrio de Romberg, escala de equilíbrio, Performance-oriented mobility assessment, Functional reach, Get-up and go e Get-up and go test. Entretanto, a estabilometria é o recurso preferencial para quantificação do equilíbrio postural, por monitorar o deslocamento do Centro de Pressão (CP) (9). O exame registra a oscilação contínua do corpo humano com o auxílio de uma plataforma de força, cujos deslocamentos ântero-posterior e médio-lateral são analisados quanto ao CP (10-12). A partir desse registro podem ser calculadas diversas variáveis relacionadas à oscilação postural $(13,14)$.

Há evidências de que desalinhamentos nas extremidades inferiores podem aumentar também o risco para queda (15). Modelos experimentais tentam elucidar a relação de possíveis alterações de pés no equilíbrio postural, com evidências de que desvios posturais podem interferir no controle postural unipodal (16) e bipodal $(17,18)$. Tsai, Yu, Mercer e Gross $(18)$ compararam três grupos com diferentes padrões morfológicos de pé por meio de mensurações estabilométricas do CP. Dos sujeitos estudados, os indivíduos de pés supinados 
tiveram aumento da velocidade média do CP. Os indivíduos com pés pronados apresentaram aumento do deslocamento do CP no sentido ântero-posterior em relação ao grupo de pés neutros. Entretanto, a repercussão das alterações morfológicas do joelho na oscilação postural não foi avaliada.

Por causa das alavancas que se formam em ortostatismo (5), a morfologia do corpo (fator estático) pode influenciar sua resposta motora (fator dinâmico) ao estímulo sensorial, alterando também o risco do indivíduo para quedas $(19,20)$. Assim, este trabalho analisou a influência da morfologia de pés e joelhos nos parâmetros estabilométricos de indivíduos jovens saudáveis.

\section{Métodos}

\section{Casuística}

O estudo realizado foi de caráter observacional transversal e contou com a participação de 55 voluntários de uma amostra de conveniência. Os indivíduos incluídos neste estudo não apresentaram: história prévia de lesões neurológicas; história de dores ou lesões nas extremidades inferiores nos últimos seis meses; uso de qualquer tipo de substância que pudesse afetar o controle postural nas últimas 24 horas (álcool ou medicamentos). Dois voluntários foram excluídos por serem praticantes de atividades físicas que requerem bom equilíbrio postural (balé e Taijiquan), restando 53 voluntários hígidos (21 [39,6\%] do sexo masculino, $26,9 \pm 5,2$ anos, $68,4 \pm 17,1 \mathrm{~kg}, 1,71 \pm 0,09 \mathrm{~m})$. O protocolo experimental foi aprovado pelo Comitê de Ética Institucional (protocolo n. 0004.0.307.000-07), de acordo com a Resolução 196/96, referente à ética em pesquisa com seres humanos. Todos os voluntários assinaram o Termo de Consentimento Livre e Esclarecido, após serem informados sobre o objetivo e natureza da pesquisa.

\section{Avaliação morfológica das extremidades inferiores}

A morfologia de pé e joelho dos voluntários foi realizada durante a avaliação postural pelo método visual não quantitativo (21). O resultado foi obtido sob concordância de dois avaliadores simultaneamente à avaliação. Os voluntários permaneceram em posição ortostática com descarga de peso, com as plantas sobre o podoscópio, para confirmação do padrão morfológico do arco longitudinal medial, com as extremidades inferiores desnudas. O padrão morfológico do joelho foi estabelecido de acordo com os critérios a seguir. Os sujeitos foram orientados a unir os maléolos mediais e, a partir deste posicionamento, observaram-se os côndilos femorais:

1) caso os côndilos femorais mediais não entrassem em contato, o joelho era classificado como varo;

2) caso os côndilos femorais se encostassem limitando o contato entre os maléolos mediais, o joelho era classificado como valgo;

3) caso os maléolos e côndilos mediais encostassem bilateralmente, os indivíduos eram classificados com joelhos neutros (valgo fisiológico).

\section{Avaliação estabilométrica}

Após a avaliação morfológica, os indivíduos cumpriam o protocolo na plataforma de força (AMTI AccuSway Plus ${ }^{\mathbb{B}}$ ) controlada pelo programa Balance Clinic ${ }^{\mathbb{B}}$. Os sujeitos se portavam em posição ortostática, descalços, com os braços ao longo do corpo, olhar fixo no horizonte e eram solicitados a permanecer o mais imóvel possível, conforme especificações da Association Française de Posturologie (22, 23). No protocolo de base aberta, os pés foram posicionados em paralelo de modo que o voluntário assumisse uma postura bipodal 
confortável. No protocolo de base fechada, os voluntários foram solicitados a unir seus maléolos mediais (ou côndilos mediais, no caso dos indivíduos com joelhos valgos). O protocolo utilizado foi de olhos abertos com base aberta (OA/BA), olhos fechados com base aberta (OF/BA), olhos abertos com base fechada $(\mathrm{OA} / \mathrm{BF})$ e olhos fechados com base fechada $(\mathrm{OF} / \mathrm{BF})$, nesta sequência. O tempo de permanência sobre a plataforma foi de 50 segundos para cada etapa e os sinais foram gravados a uma frequência de amostragem de $100 \mathrm{~Hz}(24)$.

Os sujeitos foram agrupados de acordo com as categorias de pé (neutro, cavo e plano) e joelhos (neutro, varo e valgo) separadamente. Os seguintes parâmetros estabilométricos foram calculados (13, 25): área de oscilação (da elipse do percentil $\left.95 \%, A_{95}\right)$, velocidade de oscilação média $\left(V_{A V G}\right)$ e deslocamento máximo do centroide no sentido ântero-posterior $\left(\mathrm{X}_{\mathrm{MAX}}\right)$.

\section{Análise estatística}

O coeficiente de contingência (CC, baseado no $\chi^{2}$ ) foi utilizado para verificar a associação entre os achados morfológicos de pés e joelhos. As variáveis clínicas altura, peso e idade foram comparadas por meio do teste ANOVA para os grupos de morfologias de pés e joelhos separadamente. As variáveis estabilométricas foram comparadas com ANOVA entre as morfologias de pé e joelho por protocolo. A significância estatística empregada foi de $\mathrm{p}<0,05$. Os testes foram realizados pelo programa estatístico SPSS ${ }^{\circledR}$ 16.0. Valores são exibidos como média $\pm \mathrm{DP}$ (desvio-padrão).

\section{Resultados}

O padrão de pé neutro foi encontrado em 20 (37,7\%) casos, enquanto que os padrões plano e cavo ocorreram em $27(50,9 \%)$ e $6(11,3 \%)$ casos, respectivamente. O padrão de joelho neutro também foi encontrado em 20 (37,7\%) casos, e os padrões valgo e varo ocorreram em 14 (26,4\%) e 12 (22,6\%) casos, respectivamente. Não houve associação entre os tipos morfológicos de pé e joelho na amostra estudada (CC $=0,13, \mathrm{p}=0,92)$. Não foram encontradas diferenças significativas de idade, peso e altura entre os grupos classificados pela morfologia do pé $(p=0,894 ; p=0,443 ; p=0,973$, respectivamente) ou joelho $(p=0,740$; $\mathrm{p}=0,805 ; \mathrm{p}=0,565$, respectivamente). Não houve associação significativa entre as variáveis idade, peso e altura para os grupos agrupados por morfologia de pé $(\mathrm{p}=0,560 ; \mathrm{p}=0,543 ; \mathrm{p}=0,826$, respectivamente) e joelho ( $\mathrm{p}=0,425 ; \mathrm{p}=0,426 ; \mathrm{p}=0,167$, respectivamente).

A Tabela 1 apresenta os valores das variáveis $A_{95}, V_{\text {AVG }}$ e $X_{\mathrm{MAX}}$ calculadas por protocolo e agrupadas por morfologia de pés e joelhos. Não foram encontradas diferenças estatisticamente significativas $(p>0,05)$ entres os grupos classificados por morfologia de pés para as variáveis estudadas. Diferenças significativas foram encontradas para a variável $V_{\text {AVG }}$ entres os grupos classificados por morfologia de joelho nos protocolos de BF. Indivíduos com joelho varo apresentaram menor velocidade de oscilação que os classificados como joelho neutro com OA $(p=0,009)$ e $\mathrm{OF}(\mathrm{p}=0,007)$. As variáveis $\mathrm{A}_{95}$ e $\mathrm{X}_{\mathrm{MAX}}$ não apresentaram diferenças significativas entre os indivíduos classificados por morfologia de joelho.

\section{Discussão}

Os resultados obtidos neste estudo mostram que a morfologia dos pés não altera significativamente a velocidade de oscilação, a área de oscilação ou o deslocamento ântero-posterior do CP. A literatura apresenta resultados controversos em relação ao equilíbrio postural, dependendo da morfologia do pé. Dois estudos $(16,26)$ encontraram aumento da área de excursão do CP e a atribuíram a uma menor área de contato plantar unipodal de indivíduos com pé cavo, em comparação a indivíduos com pés planos ou neutros. Essa menor área de contato plantar gera um menor bloqueio anatômico entre o aspecto medial do pé e a plataforma de força, 
como existe no pé plano e no pé neutro, e diminui a informação sensorial plantar por causa da reduzida área de contato do pé com a plataforma. Entretanto, os resultados deste estudo com apoio bipodal corroboram outro trabalho (17), que avaliou o apoio unipodal estático e não encontrou diferenças no balanço postural ântero-posterior por classificações de pé, justificando que as alterações de contato que existem entre os tipos morfológicos de pés não são suficientes para alterar a distribuição de peso na base de suporte em ortostatismo. A heterogeneidade dos resultados observada nesses estudos pode ser justificada por diferenças nos critérios de classificação da morfologia dos pés, o que reforça a necessidade de padronização na avaliação clínica e objetiva desta região.

Tabela 1 - Variáveis estabilométricas calculadas por protocolo e agrupadas por morfologia de pés e joelhos

\begin{tabular}{|c|c|c|c|c|c|c|}
\hline \multirow[t]{2}{*}{ Variável } & \multirow[t]{2}{*}{ Região } & \multirow[t]{2}{*}{ Tipo } & \multicolumn{4}{|c|}{ Protocolo } \\
\hline & & & OA/BA & $\mathrm{OA} / \mathrm{BF}$ & OF/BA & $\mathrm{OF} / \mathrm{BF}$ \\
\hline \multirow{6}{*}{$\begin{array}{l}\mathbf{A}_{95} \\
\left(10^{-2} \mathrm{~m}^{2}\right)\end{array}$} & Pé & Neutro & $1,18 \pm 0,75$ & $2,84 \pm 1,38$ & $1,50 \pm 0,85$ & $5,20 \pm 2,40$ \\
\hline & & Plano & $1,17 \pm 0,75$ & $2,85 \pm 2,11$ & $1,71 \pm 1,47$ & $5,43 \pm 3,30$ \\
\hline & & Cavo & $1,24 \pm 0,76$ & $2,80 \pm 1,76$ & $1,64 \pm 1,49$ & $4,80 \pm 2,48$ \\
\hline & Joelho & Neutro & $1,23 \pm 0,79$ & $2,88 \pm 1,93$ & $1,63 \pm 1,42$ & $5,63 \pm 3,05$ \\
\hline & & Valgo & $1,35 \pm 0,63$ & $3,15 \pm 1,93$ & $1,87 \pm 1,22$ & $5,55 \pm 3,01$ \\
\hline & & Varo & $0,88 \pm 0,70$ & $2,39 \pm 1,28$ & $1,33 \pm 0,92$ & $4,12 \pm 2,02$ \\
\hline \multirow{6}{*}{$\begin{array}{l}\mathbf{V}_{\mathbf{A V G}} \\
\left(10^{-2} \mathrm{~m} / \mathrm{s}\right)\end{array}$} & Pé & Neutro & $10,20 \pm 5,92$ & $10,74 \pm 6,32$ & $10,32 \pm 5,83$ & $10,21 \pm 5,70$ \\
\hline & & Plano & $9,47 \pm 5,50$ & $9,82 \pm 5,03$ & $9,08 \pm 5,26$ & $10,06 \pm 4,56$ \\
\hline & & Cavo & $10,59 \pm 5,66$ & $10,69 \pm 3,92$ & $10,29 \pm 4,22$ & $9,65 \pm 4,40$ \\
\hline & Joelho & Neutro & $10,48 \pm 6,72$ & $11,61 \pm 6,36$ & $10,56 \pm 6,33$ & $11,38 \pm 5,82$ \\
\hline & & Valgo & $10,45 \pm 3,91$ & $10,59 \pm 3,52$ & $10,23 \pm 3,31$ & $10,24 \pm 3,14$ \\
\hline & & Varo & $7,81 \pm 4,08$ & $6,86 \pm 3,04 *$ & $7,11 \pm 4,18$ & $6,92 \pm 2,77 *$ \\
\hline \multirow{6}{*}{$\begin{array}{l}\mathbf{X}_{\mathrm{MAX}} \\
\left(10^{-2} \mathrm{~m}\right)\end{array}$} & Pé & Neutro & $0,53 \pm 0,21$ & $1,06 \pm 0,28$ & $0,59 \pm 0,30$ & $1,51 \pm 0,47$ \\
\hline & & Plano & $0,53 \pm 0,24$ & $1,15 \pm 0,45$ & $0,65 \pm 0,38$ & $1,68 \pm 0,53$ \\
\hline & & Cavo & $0,64 \pm 0,34$ & $1,08 \pm 0,24$ & $0,77 \pm 0,68$ & $1,86 \pm 0,45$ \\
\hline & Joelho & Neutro & $0,56 \pm 0,25$ & $1,15 \pm 0,39$ & $0,66 \pm 0,42$ & $1,65 \pm 0,41$ \\
\hline & & Valgo & $0,58 \pm 0,25$ & $1,03 \pm 0,31$ & $0,64 \pm 0,46$ & $1,59 \pm 0,64$ \\
\hline & & Varo & $0,46 \pm 0,18$ & $1,11 \pm 0,42$ & $0,60 \pm 0,26$ & $1,65 \pm 0,56$ \\
\hline
\end{tabular}

${ }^{*} p<0,05$; comparado com Neutro. Valores são exibidos como média \pm DP. $\mathrm{OA}=\mathrm{olhos}$ abertos; $\mathrm{OF}=\mathrm{olhos}$ fechados; $\mathrm{BA}=\mathrm{base}$ aberta; $\mathrm{BF}=$ base fechada. 
Considerando que a habilidade de realizar compensações posturais é essencial para prevenir lesões, os resultados obtidos sugerem que as alterações morfológicas de pé não modificam significativamente a oscilação corporal e, consequentemente, não aumentam a instabilidade corporal e o risco de lesões por queda. Entretanto, ainda não está claro se uma maior ou menor oscilação é benéfica ou prejudicial (17); variabilidade aumentada pode conduzir à fadiga por contração muscular prolongada ou repetitiva, mas também pode sugerir maior flexibilidade e capacidade adaptativa postural para responder às perturbações repentinas. Assim, os resultados deste trabalho sugerem que a correção de possíveis alterações morfológicas de pé (plano, cavo) não seja necessária para a redução do risco de quedas em indivíduos jovens com déficits de equilíbrio postural. Esse desfecho deve ser avaliado em estudos prospectivos em outras populações de risco para quedas (como os idosos), para verificação da generalização dessa hipótese.

Neste trabalho, observou-se a redução da velocidade de oscilação em indivíduos com joelho varo quando a base de suporte foi diminuída, nas condições OA e OF. Não foram encontrados estudos referentes à comparação estabilométrica dos tipos de joelho em apoio bipodal. Há evidências de que a morfologia do joelho altera a biomecânica da extremidade inferior em apoio unipodal com OF, pois indivíduos com joelhos em valgo ou varo dependem mais da função de controle das articulações subtalar e mediotársica para o controle postural dinâmico (18). O fato de o joelho apresentar menos graus de liberdade que o tornozelo pode explicar tal observação. Assim, os resultados deste trabalho sugerem que a intervenção terapêutica para correção/compensação do joelho varo seja considerada para indivíduos jovens com déficits de equilíbrio postural. Entretanto, o que representa essa redução na velocidade de oscilação e como ela afeta o risco para quedas em outras populações ainda precisa ser determinado.

Não há na literatura um método padrão-ouro para a classificação dos tipos de pés e joelhos (27-31). Optou-se pela avaliação postural visual (com concordância simultânea de dois avaliadores), por ser a maneira mais difundida e utilizada na prática clínica e por apresentar boa correlação com achados radiográficos (21, 32, 33). Neste estudo foram desconsiderados, inicialmente, os dados referentes a valgo e varo do retropé e antepé, pois a relação destes desvios posturais é menos integrada às avaliações clínicas nos ambulatórios fisioterápicos em relação aos dados analisados.

\section{Conclusão}

Este estudo mostrou que a morfologia do pé não altera significativamente a velocidade de deslocamento, a área de oscilação ou o deslocamento ântero-posterior. Entretanto, a redução da base de suporte reduz a velocidade de oscilação em indivíduos com joelho varo.

\section{Referências}

1. Teasdale N, Simoneau M. Attentional demands for postural control: the effects of aging and sensory reintegration. Gait Posture. 2001;14(3):203-10.

2. Murphy DF, Connolly DAJ, Beynnon BD. Risk factors for lower extremity injury: a review of the literature. Br J Sports Med. 2003;37(1):13-29.

3. Hijmans MJ, Geertzen BHJ, Dijkstra UP, Postema K. A systematic review of the effects of shoes and other ankle or foot appliances on balance in older people and people with peripheral nervous system disorders. Gait Posture. 2007;25(2):316-23.

4. Redfern SM, Yardley L, Bronstein AM. Visual influences on balance. J Anxiety Disord. 2001;15:81-94.

5. Smith KL, Weiss LE, Don Lehmkuhl L. Cinesiologia clínica de Brunnstrom. São Paulo: Manole; 1997.

6. Bouisset S. Posture, dynamic stability, and voluntary movement. Neurophysiol Clin. 2008;38(6):345-62. 
7. Redfern SM, Jennings RJ, Martin C, Furman MJ. Attention influences sensory integration for postural control in older adults. Gait Posture. 2001;14(3):211-6.

8. Freitas VE, Py L, Neri LA, Cançado XAA, Gorzoni LM, Rocha MS. Tratado de geriatria e gerontologia. Rio de Janeiro: Guanabara Koogan; 2002.

9. Monsell EM, Furman JM, Herdman SJ, Konrad HR, Shepard NT. Computerized dynamic platform posturography. Otolaryngol Head Neck Surg. 1997;117(4):394-8.

10. Oliveira LF, Imbiriba LA, Garcia MAC. Índice de estabilidade para avaliação do equilíbrio postural. Brazil J Biomech. 2000;(1)1:33-8.

11. Mochizuki L, Amadio CA. Aspectos biomecânicos da postura ereta: a relação entre o centro de massa e o centro de pressão. Rev Port Cien Desp. 2003;3(3):77-83.

12. Rougier PR. What insights can be gained when analysing the resultant centre of pressure trajectory? Neurophysiol Clin. 2008;38(6):363-73.

13. Raymakers JA, Samson MM, Verhaar HJJ. The assessment of body sway and the choice of the stability parameter(s). Gait Posture. 2005;21(1):48-58.

14. Chaudhry H, Bukiet B, JI Z, Findley T. Measurement of balance in computer posturography: comparison of methods - A brief review. J Bodyw Mov Ther. [serial on the internet] 2008 [cited 200915 jun]. Available from: http://dx.doi.org/10.1016/j.jbmt.2008.03.003

15. Menz HB, Morris ME, Lord SR. Foot and ankle characteristics associated with impaired balance and functional ability in older people. J Gerontol A Biol Sci Med Sci. 2005;60(12):1546-52.

16. Hertel J, Gay RM, Denegar RC. Differences in postural control during single-leg stance among healthy individuals with different foot types. J Athl Train. 2002;37(2):129-32.

17. Cote PK, Brunet EM, Gansneder BM, Shultz SJ. Effects of pronated and supinated foot postures on static and dynamic postural stability. J Athl Train. 2005 Mar;40(1):41-6.

18. Tsai LC, Yu B, Mercer VS, Gross MT. Comparison of different structural foot types for measures of standing postural control. J Orthop Sports Phys Ther. 2006;36(12):942-53.

19. Günther M, Grimmer S, Siebert T, Blickhan R. All leg joints contribute to quiet human stance: A mechanical analysis. J Biomech. 2009;42(16):2739-46.

20. Hsu WL, Scholz JP, Schöner G, Jeka JJ, Kiemel T. Control and estimation of posture during quiet stance depends on multijoint coordination. J Neurophysiol. 2007;97(4):3024-35.

21. Razeghi M, Batt ME. Foot type classification: a critical review of current methods. Gait Posture. 2002;15(3):282-91.

22. Kapteyn TS, Bles W, Njiokiktjien CJ, Kodde L, Massen CH, Mol JMF. Standardization in Platform Stabilometry being a Part of Posturography. Agressologie. 1983;24(7):321-6.

23. Bizzo G, Guillet N, Patat A, Gagey P. Specifications for building a vertical force platform designed for clinical stabilometry. Med Biol Eng Comput. 1985;23(5):474-6.

24. Prieto ET, Myklebust BJ, Myklebust MB. characterization and modeling of postural steadiness in the elderly: a review. IEEE Trans Rehabil Eng.1993;1:26-33.

25. Cornilleau-Peresa V, Shabana N, Droulez J, Goh JCH, Lee GSM, Chew PTK. Measurement of the visual contribution to postural steadiness from the COP movement: methodology and reliability. Gait Posture. 2005;22(2):96-106.

26. Wong L, Hunt A, Burns J, Crosbie J. Effect of foot morphology on center-of-pressure excursion during barefoot walking. J Am Podiatr Med Assoc. 2008;98(2):112-7. 
27. Mathieson I, Upton D, Prior TD. Examining the validity of selected measures of foot types. J Am Podiatr Med Assoc. 2004;94(3):275-81.

28. Redmond AC, Crosbie J, Ouvrier RA. Development and validation of a novel rating system for scoring standing foot posture: the Foot Posture Index. Clin Biomech. 2006;21(1):89-98.

29. Wrobel JS, Armstrong DG. Reliability and validity of current physical examination techniques of the foot and ankle. J Am Podiatr Med Assoc. 2008;98(3):197-206.

30. Nikolaidou ME, Boudolos KD. A footprint-based approach for the rational classification of foot types in young schoolchildren. Foot. 2006;16(2):82-90.

31. Keenan AM, Redmond AC, Horton M, Conaghan PG, Tennant A. The foot posture index: rasch analysis of a novel, foot-specific outcome measure. Arch Phys Med Rehabil. 2007;88(1):88-93.

32. Song J, Hillstrom HJ, Secord D, Levitt J. Foot type biomechanics. comparison of planus and rectus foot types. J Am Podiatr Med Assoc. 1996;86(1):16-23.

33. Lamm BM, Mendicino RW, Catanzariti AR, Hillstrom HJ. Static rearfoot alignment: a comparison of clinical and radiographic measures. J Am Podiatr Med Assoc. 2005;95(1):26-33.

Recebido: 25/08/2009

Received: 08/25/2009

Aprovado: $23 / 03 / 2010$

Approved: 03/23/2010

Revisado: 05/05/2010

Reviewed: 05/05/2010 